\title{
Cryptococcus gattii pneumonia
}

\author{
Kerri A. Johannson MD, Shaunna M. Huston RN BSc, Christopher H. Mody MD, Warren Davidson MD MHSc
}

A 27-year-old previously healthy woman presented to the emergency department in Calgary, Alberta, with shortness of breath, muscle aches and a nonproductive cough that had lasted for 48 hours. During the previous two weeks, the patient had experienced a loss of appetite, fatigue, fever and chills. She reported smoking tobacco and marijuana daily and drinking 12 alcoholic drinks per week; she did not use injection drugs. She took no medications and had no known allergies. Her travel history included a trip to Puerto Escondido in Oaxaca, Mexico, one year earlier and a trip to Vancouver Island, British Columbia, 36 months before presentation. While on Vancouver Island, the patient had spent time hiking and camping in the forested areas of Parksville and Cathedral Grove.

Physical examination showed oxygen saturation of $93 \%$ while the patient was breathing ambient air (atmospheric pressure $660 \mathrm{~mm} \mathrm{Hg}$ ), and scattered inspiratory crackles were heard on chest auscultation. The rest of the examination was unremarkable. Laboratory investigations showed a white blood cell count of 21.4 (normal $4.0-11.0) \times 10^{9} / \mathrm{L}$, with 17.3 (normal $\left.2.0-8.0\right) \times$ $10^{\circ} / \mathrm{L}$ neutrophils and 601 (normal $\left.150-400\right) \times$ $10^{9} / \mathrm{L}$ platelets. Radiography of the patient's chest showed multiple pulmonary nodules with mass-like areas of opacification (Figure 1). Computed tomography showed extensive patchy nodular areas of consolidation with early cavitations, predominantly in the upper lobes of the lungs (Figure 2). The differential diagnosis included atypical bacterial and fungal infections, septic emboli, metastatic disease and granulomatosis with polyangiitis.

Stained tissue samples from endobronchial and transbronchial biopsies of the right lower lobe of the patient's lung were consistent with Cryptococcus species, and bronchoalveolar lavage cultures were positive for Cryptococcus gattii. The organism was genotyped and found to be strain VGIIa, most frequently isolated from Vancouver Island. Tests of the patient's serum showed cryptococcal antigen at a titre of 1:256. We prescribed oral fluconazole to be taken daily, with follow-up as an outpatient.

We assessed the patient's immune function. Testing for HIV was negative, and her total serum level of immunoglobulin $\mathrm{G}$ ( $\mathrm{IgG}$ ) was normal. The patient's IgG subclass levels (subclasses 1, 2, 3 and 4), diphtheria, tetanus and pneumococcal serologies, and complement levels were normal, as were her isohemagglutinin anti-A (32, normal 8-2048) and anti-B (16, normal 8-512) titres. After the patient had recovered, flow cytometry showed normal percentages and numbers of $\mathrm{CD} 3+\mathrm{T}$ cells, CD4+ T cells, CD8+ T cells, CD19+ B cells and CD3-/ CD16/56+ natural killer (NK) cells. Lymphocyte transformation to phytohemagglutinin, concanavalin A, pokeweed mitogen and Staphylococcus aureus were all normal, as assessed by the expression of Ki67 and proliferating cell nuclear antigen. The patient's serum level of immunoglobulin E was initially elevated (258.5 [normal 0-160] kIU/L), but normalized within three months of the start of treatment.

At two years' follow-up, tests of the patient's serum were negative for cryptococcal antigen, and fluconazole was subsequently discontinued. Spirometry showed that reversible airflow obstruction remained, and chronic parenchymal opacities were visible on radiography of the chest. We have now followed our patient for four years and have seen no evidence of the recurrence of disease.
Competing interests: Christopher Mody has been reimbursed for travel and accommodations by the Canadian Institutes of Health Research and the British Columbia Centre for Disease Control. Warren Davidson has consulted for AstraZeneca, Boehringer Ingelheim, GlaxoSmithKline and Novartis; he has received grants from GlaxoSmithKline and Novartis; he has received payment for lectures from AstraZeneca, Boehringer Ingelheim, GlaxoSmithKline, Novartis and Pfizer; and he has been reimbursed for travel and accomodations by Boehringer Ingelheim. No other competing interests were declared.

This article has been peer reviewed.

Correspondence to: Warren Davidson, wdavidso@ucalgary.ca

CMAJ 2012. DOI:10.1503 /cmaj.111346

\section{KEY POINTS}

- Cryptococcus gattii is an endemic fungus causing pulmonary or central nervous system disease, typically in immunocompetent hosts.

- History of travel, even if several years earlier, to an area endemic for C. gattii should raise suspicion for this etiologic agent; genotyping may help to identify the geographic source of the organism.

- Because colonization with Cryptococcus can occur, a diagnosis of infection requires evidence of pathology or dissemination.

- Infection with C. gattii should be treated with antifungal agents for at least 6 months. 


\section{Discussion}

Cryptococcus species are environmental fungi, acquired through inhalation, that can cause lifethreatening infections of the pulmonary and central nervous systems. Of the 37 known species, Cryptococcus neoformans and C. gattii are the most commonly identified pathogens, causing a spectrum of disease that includes fever, cough, pneumonia, meningoencephalitis, widespread dissemination and even death. Cryptococcus neoformans usually affects people with HIV, but C. gattii primarily causes disease in immunocompetent patients. The major risk factor for acquiring $C$. gattii infection is a history of living in or travel to an endemic area. Other risk factors include age greater than 50 years, smoking, use of corticosteroids, and history of cancer or chronic lung disease. ${ }^{1}$

The long incubation period of $C$. neoformans (median 110 months) suggests a period of dormancy before reactivation. ${ }^{2}$ However, the median incubation period for all strains of Vancouver Island $C$. gattii is reported to be 6-7 (range 2-11) months, which is more consistent with a primary infection. ${ }^{2}$ Our patient's case highlights the potential for an unusually prolonged incubation period between exposure to C. gattii in an endemic area and clinical presentation.

\section{Endemic areas in North America}

On Vancouver Island, $C$. gattii is primarily found in the air, and on and under the canopy of trees in the coastal Douglas fir regions of Parksville and Cathedral Grove. ${ }^{3}$ The number of people with $C$. gattii infections on Vancouver Island increased sharply between 1999 and 2002, prompting heightened interest in the epidemiology and clinical manifestations of this disease. Between 1999 and 2010, 281 cases were reported on Vancouver Island, with a mortality of about 9\%. ${ }^{1,4}$ Most of these cases affected immunocompetent patients, and $75 \%$ of cases involved pulmonary infections.

Since 2004, C. gattii has surfaced as a pathogen in the Pacific Northwest of the United States, in close proximity to Vancouver Island. Between 2004 and 2010, 60 cases were reported in humans, with 25\% mortality. ${ }^{5}$ All cases reported to 2004 involved patients who had lived on or travelled to Vancouver Island; however, MacDougall and colleagues ${ }^{6}$ subsequently reported five cases of $C$. gattii in patients with no identifiable exposures on Vancouver Island during the previous year. The patients lived on the southwestern coastal mainland of BC, or in coastal Washington or Oregon in the US. Four of the five had underlying immunodeficiencies. Cryptococcus has also caused infections in Mexico; one retrospective study identified 8 of 72 cases of cryptococcal infection as caused by $C$. gattii. $^{7}$

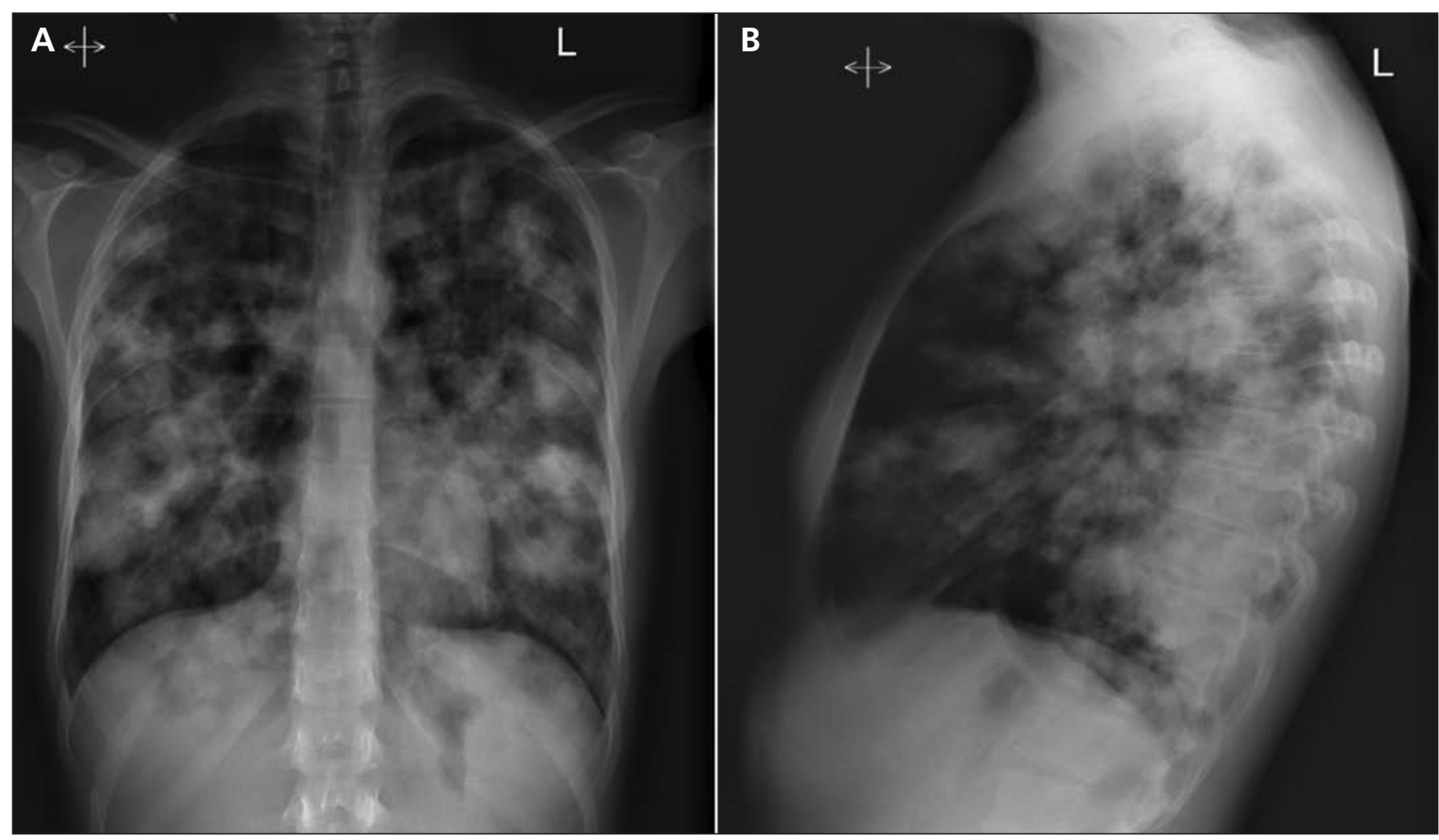

Figure 1: Radiographic image of the posteroanterior (A) and lateral (B) chest of a 27-year-old woman presenting with shortness of breath, muscle aches and cough. Multiple pulmonary nodules with mass-like areas of opacification can be seen. 


\section{Diagnosis and management}

Pulmonary manifestations of infection with C. gattii include multifocal airspace disease, solitary nodules, pleural effusions, endobronchial lesions and multiple cavitary nodules. Hypoxemia and severe sepsis are rare. Because people can be colonized with Cryptococcus, a diagnosis of infection requires evidence of pathology (radiographic, clinical or pathologic) or dissemination (positive serum Cryptococcal antigen titre). If the serum level of cryptococcal antigen is elevated, and certainly if neurologic symptoms are present, then a lumbar puncture should be done to exclude involvement of the central nervous system.

The 2011 statement from the American Thoracic Society outlines treatment recommendations based on the extent and location of disease. ${ }^{8}$ Azole compounds, such as fluconazole, are recommended for treating isolated pulmonary infection, whereas treatment with amphotericin B deoxycholate plus flucytosine is reserved for disseminated disease or involvement of the central nervous system. ${ }^{8}$ Treatment should last for at least six months, with the length of therapy tailored to the extent of disease and clinical response.

\section{Immunocompetency}

Although C. gattii is thought to mostly affect immunocompetent patients, some patients have been found to have immune defects. ${ }^{6}$ For this reason, we assessed our patient for a possible immunodeficiency. Our patient had normal IgG isotype levels and normal diphtheria and tetanus serology, suggesting an adequate functional humoral immune response (IgG subclass 1); thus, it was unlikely that she had an immunodeficiency or isotype deficiencies. The results of pneumococcal serology were normal, suggesting adequate IgG subclass 2 response to polysaccharide antigens. Our patient's normal isohemagglutinin titres suggested adequate $\operatorname{IgG}$ and immunoglobulin $\mathbf{M}$ responses to polysaccharide antigens. Levels of total complement and complement components 3 and 4 were normal, suggesting adequate functional response of the classical pathway. Normal levels of circulating lymphocyte subsets and normal lymphocyte transformation without evidence of clonal aberrancy suggested that there was no defect in cellmediated immunity. The patient's immunoglobulin E level was initially elevated but normalized within three months of treatment, making hyperimmunoglobulin E syndrome (Job syndrome) unlikely. The negative results of our workup for immunodeficiency lends further support to the current understanding that $C$. gattii mostly affects people with intact immunity.

\section{Geographic source of our patient's infection}

Our patient lived in Calgary, but she had hiked and camped in the forested areas of Parksville and Cathedral Grove, BC, which are endemic for C. gattii. Of the $C$. gattii cases identified in BC from 1999 to 2007, 86.3\% were the VGIIa strain, ${ }^{6}$ as was the etiologic agent in our patient's infection. Our patient had also visited Mexico; as mentioned previously, one retrospective study identified 8 of 72 cryptococcal cases in Mexico as having been caused by $C$. gattii, two of which had the VGII genotype. ${ }^{7}$ Unfortunately, information regarding VGIIa in these two isolates is unavailable. However, the isolates were from areas other than where our patient had travelled, and as yet there are no reports of the VGIIa strain in the area in Mexico where our patient visited. Thus, the geographic distribution of genotypes makes it highly unlikely that she acquired the infection in Mexico.

MacDougall and colleagues propose that offisland cases of $C$. gattii infection may be due to fungal elements being carried across the Strait of Georgia from Vancouver Island to the mainland via airborne or anthropogenic dispersal. ${ }^{6}$ However, no infections with the VGIIa strain of C. gattii have been shown to be acquired in Calgary, and the distance between Vancouver Island and Calgary $(>1000 \mathrm{~km}$, separated by the Rocky and Coast Mountains) makes local acquisition in the case of our patient unlikely.

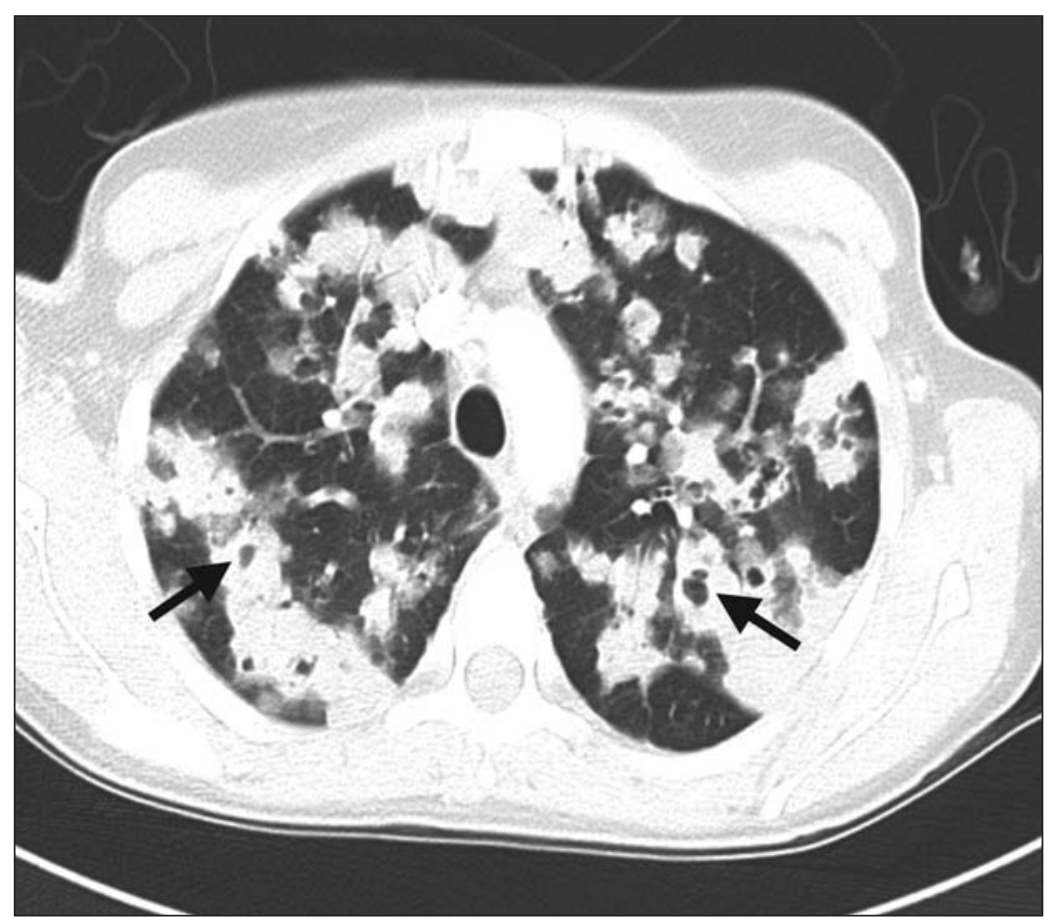

Figure 2: Enhanced computed tomography scan of the patient's chest at presentation. Bilateral parenchymal infiltrates with areas of cavitation are prominent in the upper lobes of the lungs (arrows). 
Physicians caring for patients who have visited an area endemic for $C$. gattii should be aware of the potential for the disease to develop after a prolonged incubation and include it in their differential diagnosis of pneumonia in an immunocompetent patient.

\section{References}

1. Bartlett KH, Cheng P-Y, Duncan C, et al. A decade of experience: Cryptococcus gattii in British Columbia. Mycopathologia 2012;173:311-9.

2. MacDougall L, Fyfe M. Emergence of Cryptococcus gattii in a novel environment provides clues to its incubation period. J Clin Microbiol 2006;44:1851-2.

3. Kidd SE, Hagen F, Tscharke RL, et al. A rare genotype of Cryptococcus gattii caused the cryptococcus outbreak on Vancouver Island (British Columbia, Canada). Proc Natl Acad Sci U S A 2004;101:17258-63.

4. Galanis E, MacDougall L. Epidemiology of Cryptococcus gattii, British Columbia, Canada, 1999-2007. Emerg Infect Dis 2010; 16:251-7.

5. Emergence of Cryptococcus gattii — Pacific Northwest 2004 2010. MMWR Morb Mortal Wkly Rep 2010;59:865-8.

6. MacDougall L, Kidd S, Galanis E, et al. Spread of Cryptococcus gattii in British Columbia, Canada, and detection in the Pacific Northwest, USA. Emerg Infect Dis 2007;13:42-50.

7. Olivares LR, Martinez KM, Cruz RM, et al. Genotyping of Mexican Cryptococcus neoformans and C. gattii isolates by PCR-fingerprinting. Med Mycol 2009;47:713-21.

8. Limper AH, Knox KS, Sarosi GA, et al.; American Thoracic Society Fungal Working Group. An Official American Thoracic Society statement: treatment of fungal infections in adult and pulmonary critical care patients. Am J Respir Crit Care Med 2011;183:96-128.
Affiliation: From the Department of Internal Medicine, Division of Respirology, University of Calgary, Calgary, Alta.

Contributors: Kerri Johannson and Warren Davidson conceived the idea for the report. Kerri Johannson, Christopher Mody and Warren Davidson interpreted the data and drafted the article. Shaunna Huston, Christopher Mody and Warren Davidson acquired and analyzed the data. All of the authors revised the article for important intellectual content and approved the final version submitted for publication.

Acknowledgements: The authors thank the patient for consenting to the publication of this case and these images, as well as Dr. Karen Bartlett for her helpful commentary in reviewing this manuscript.

The section Cases presents brief case reports that convey clear, practical lessons. Preference is given to common presentations of important rare conditions, and important unusual presentations of common problems. Articles start with a case presentation (500 words maximum), and a discussion of the underlying condition follows (1000 words maximum). Visual elements (e.g., tables of the differential diagnosis, clinical features or diagnostic approach) are encouraged. Written consent from patients for publication of their story is a necessity and should accompany submissions. See information for authors at www.cmaj.ca.

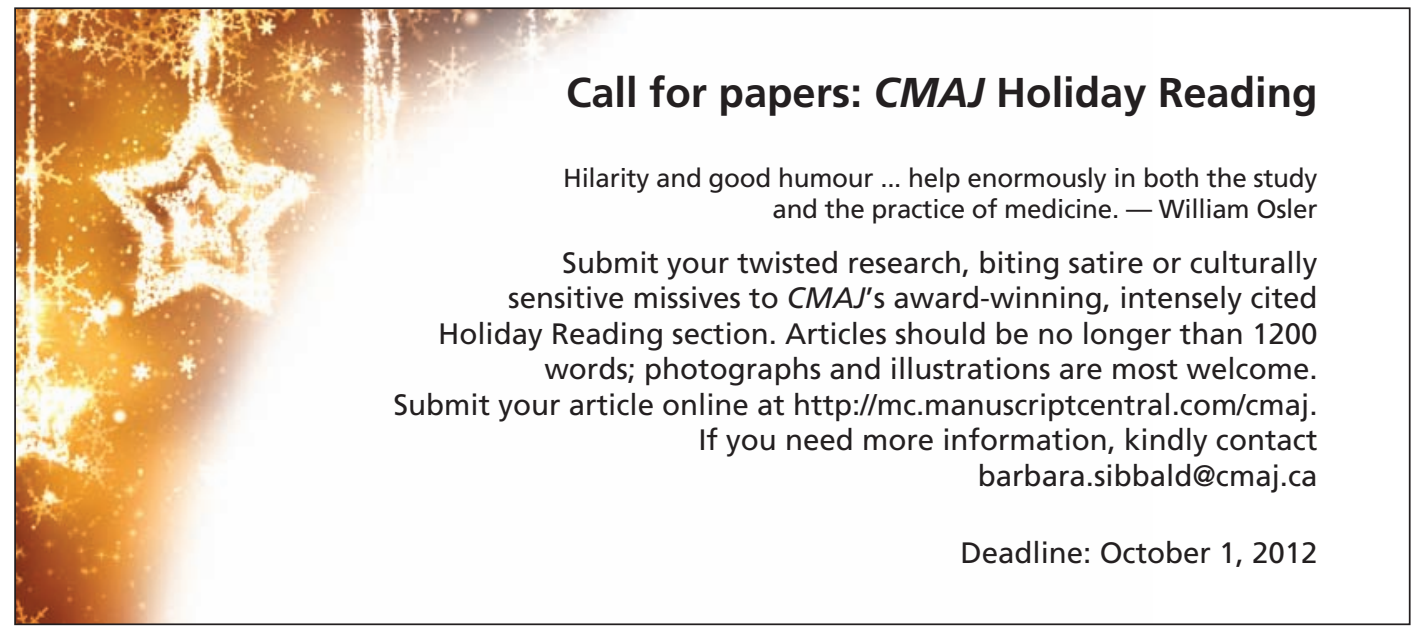

